\title{
The Morse minimal system is finitarily Kakutani equivalent to the binary odometer
}

\author{
by
}

\author{
Mrinal Kanti Roychowdhury (Denton, TX) and \\ Daniel J. Rudolph (Fort Collins, CO)
}

\begin{abstract}
Two invertible dynamical systems $(X, \mathfrak{A}, \mu, T)$ and $(Y, \mathfrak{B}, \nu, S)$, where $X$ and $Y$ are Polish spaces and Borel probability spaces and $T, S$ are measure preserving homeomorphisms of $X$ and $Y$, are said to be finitarily orbit equivalent if there exists an invertible measure preserving mapping $\phi$ from a subset $X_{0}$ of $X$ of measure one onto a subset $Y_{0}$ of $Y$ of full measure such that

(1) $\left.\phi\right|_{X_{0}}$ is continuous in the relative topology on $X_{0}$ and $\left.\phi^{-1}\right|_{Y_{0}}$ is continuous in the relative topology on $Y_{0}$,

(2) $\phi\left(\operatorname{Orb}_{T}(x)\right)=\operatorname{Orb}_{S}(\phi(x))$ for $\mu$-a.e. $x \in X$.

$(X, \mathfrak{A}, \mu, T)$ and $(Y, \mathfrak{B}, \nu, S)$ are said to be finitarily evenly Kakutani equivalent if they are finitarily orbit equivalent by a mapping $\phi$ for which there are measurable subsets $A$ of $X$ and $B=\phi(A)$ of $Y$ with $\phi$ an isomorphism of $T_{A}$ and $T_{B}$.

It is shown here that the Morse minimal system and the binary odometer are finitarily evenly Kakutani equivalent.

1. Introduction. Finitary or almost continuous dynamics lies at the interface of topological and measurable dynamics. Most naturally arising dynamical systems come endowed with both a topological and measure preserving structure. We begin by defining what it means for two spaces to be finitarily isomorphic.
\end{abstract}

Definition 1.1. Suppose $(X, \mathfrak{A}, \mu)$ and $(Y, \mathfrak{B}, \nu)$ are Borel probability spaces where $X, Y$ are each Polish topological spaces. A map $\phi$ is called finitary or almost continuous from $X$ to $Y$ if there exist subsets $X_{0} \subseteq X$ and $Y_{0} \subseteq Y$ each of full measure and $\phi: X_{0} \rightarrow Y_{0}$ is measure preserving and continuous in the relative topologies on these subsets.

2000 Mathematics Subject Classification: 28D05, 37A20.

Key words and phrases: Morse minimal system, binary odometer, finitary equivalence, Kakutani equivalence.

This work supported in part by NSF grant DMS0618030. 
If $\phi$ is one-to-one and $\phi^{-1}$ is also almost continuous, then $\phi$ is called an almost continuous or finitary isomorphism of these two spaces.

It is an exercise that all uncountable Polish spaces endowed with a nonatomic Borel probability measure are finitarily isomorphic. If one has dynamics on each of the spaces in the form of a measure preserving homeomorphism then one can seek to show these dynamical systems are finitarily equivalent in a variety of forms.

Definition 1.2. Two invertible dynamical systems $(X, \mathfrak{A}, \mu, T)$ and $(Y, \mathfrak{B}, \nu, S)$, where $X$ and $Y$ are Polish spaces and Borel probability spaces and $T, S$ are measure preserving homeomorphisms of $X$ and $Y$, are said to be finitarily orbit equivalent if there exists an invertible measure preserving mapping $\phi$ from a subset $X_{0}$ of $X$ of measure one onto a subset of $Y_{0}$ of $Y$ of full measure such that

(1) $\left.\phi\right|_{X_{0}}$ is continuous in the relative topology on $X_{0}$ and $\left.\phi^{-1}\right|_{Y_{0}}$ is continuous in the relative topology on $Y_{0}$,

(2) $\phi\left(\operatorname{Orb}_{T}(x)\right)=\operatorname{Orb}_{S}(\phi(x))$ for $\mu$-a.e. $x \in X$.

The finitary theory began in the study of isomorphism of Bernoulli shifts and Markov chains and this background can be found in [KS2], [KS3]. More recently the broad study of finitary orbit equivalence was begun by Hamachi and Keane $[\mathrm{HK}]$. They described how to construct a finitary orbit equivalence mapping between the binary odometer and the ternary odometer. For recent development of finitary theory see [HKR], [R1], [R2], [RR1], [RR2]. In this sense the work here sits between these two areas as even Kakutani equivalence is a natural equivalence relation stronger than orbit equivalence and weaker than isomorphism. Our work follows the basic schema laid down by Keane and Hamachi.

Definition 1.3. $(X, \mathfrak{A}, \mu, T)$ and $(Y, \mathfrak{B}, \nu, S)$ are said to be finitarily evenly Kakutani equivalent if they are finitarily orbit equivalent by a mapping $\phi$ for which there are measurable subsets $A$ of $X$ and $B=\phi(A)$ of $Y$ and $T_{A} \cong T_{B}$ by $\phi$.

If $\phi$ is only an invertible measure preserving mapping then $T$ and $S$ are said to be evenly Kakutani equivalent. This is a much studied area (see $[$ ORW]). Previously we have shown that all uniform odometers are finitarily Kakutani equivalent. Here we show that the binary odometer and the Morse minimal system, with its unique invariant measure, are finitarily Kakutani equivalent. In the context of such homeomorphisms of Cantor sets the topological orbit equivalence theory of Giordano, Putnam and Skau applies and in fact the binary odometer and the Morse system are homeomorphically orbit equivalent. We ask whether this can be strengthened to a homeomorphic Kakutani equivalence and conjecture it cannot. 
We remind the reader of the construction of the Morse system as we will need a basic understanding. One first constructs the classical Morse sequence $w$ by iterating the substitution $0 \rightarrow 01$ and $1 \rightarrow 10$ on the symbol 0 . This is a sequence in $\{0,1\}^{\mathbb{N}}$. The Morse minimal set is the "orbit closure" of this sequence in $\{0,1\}^{\mathbb{Z}}$ by which we mean all doubly infinite sequences for which every finite subword occurs somewhere as a subword of $w$.

The Morse minimal set $X$ is well known to be uniquely ergodic. The Morse minimal set together with its uniquely ergodic complete Borel probability measure $\mu$ and the shift map $\sigma$ is called the Morse minimal system. The Morse minimal system and the binary odometer are very closely related. The former is in fact a two-point extension of the latter. This is enough to show that the two systems are measurably evenly Kakutani equivalent as it implies the Morse system is "loosely Bernoulli" in the sense of [ORW]. In this paper we show

MAIn TheORem. The Morse minimal system and the binary odometer are finitarily evenly Kakutani equivalent.

\section{Basic definitions, lemmas and propositions on the Morse} minimal system. In this section we lay out the structure we will need in the Morse minimal system.

Let $B=b_{1} \cdots b_{n} \subseteq\{0,1\}^{n}$ be an $n$-block. The block $\bar{B}=\bar{b}_{1} \cdots \bar{b}_{n}$ is defined by setting $\bar{b}_{i}=0$ if $b_{i}=1$ and $\bar{b}_{i}=1$ if $b_{i}=0, i=1, \ldots, n$. We call $\bar{B}$ the flip of $B$.

Let $\zeta$ be the Morse substitution on $\{0,1\}$ defined by $\zeta(0)=01$ and $\zeta(1)=10$. Now define the sequence of blocks $u_{k}$ and $\bar{u}_{k}$ such that $u_{k}=\zeta^{k}(0)$ and $\bar{u}_{k}=\zeta^{k}(1), k \geq 0$. Thus $u_{k+1}=u_{k} \bar{u}_{k}$ and the Morse sequence is that sequence whose first $2^{k}$ symbols are the word $u_{k}$.

Proposition 2.1. For each $w^{\prime} \in X$ there exists a unique partition of $\mathbb{Z}$ into intervals of length $2^{k+1}$ so that the subword of $w^{\prime}$ on each interval of this partition is either $u_{k} \bar{u}_{k}$ or $\bar{u}_{k} u_{k}$.

Proof. We are sure this is a known result. The existence of this partition is claimed without argument in [CKL]. We give a brief proof of existence as well as uniqueness. To begin, notice this is true of the Morse sequence $w$ itself as on each interval $\left[j 2^{k+1}+1,(j+1) 2^{k+1}\right]$ one sees either the subword $u_{k} \bar{u}_{k}$ or $\bar{u}_{k} u_{k}$. Let $w^{\prime} \in X$ and consider the subinterval $[-n, n]$ in $\mathbb{Z}$. Since the subword $w_{[-n, n]}^{\prime}=w_{-n}^{\prime} w_{-n+1}^{\prime} \cdots w_{n}^{\prime}$ of $w^{\prime}$ occurs somewhere in $w$, the word $w_{[-n, n]}^{\prime}$ can be partitioned into occurrences of such pairs, with of course possible partial subwords at the two ends. Index 0 of $w^{\prime}$ occurs at some position in some pair. Let $t_{n}$ be the distance of the 0th position of $w^{\prime}$ from the right end point of the pair containing it. Then $0 \leq t_{n}<2^{k+1}$. As 
$\left\{t_{n}: n \in \mathbb{N}\right\}$ is a bounded sequence of natural numbers, it has a constant subsequence $\left\{t_{n_{i}}\right\}_{i \geq 1}$, i.e. $t_{n_{i}}=t$ (constant) for all $i \geq 1$. For $j \in \mathbb{Z}$ let

$$
I_{j}=\left[t+(j-1) 2^{k+1}+1, \ldots, t+j 2^{k+1}\right] .
$$

It follows that the subword of $w^{\prime}$ on each block $I_{j}$ must be one of the two choices $u_{k} \bar{u}_{k}$ or $\bar{u}_{k} u_{k}$. Now we show that the partition is unique.

If possible suppose $w^{\prime} \in X$ and there are two partitions

$$
P=\left\{\cdots<I_{-1}<I_{0}<I_{1}<\cdots\right\}, \quad P^{\prime}=\left\{\cdots<J_{-1}<J_{0}<J_{1}<\cdots\right\},
$$

where each $I_{k}$ and $J_{k}$ is an interval of length $2^{k+1}$ and

$$
w_{I_{j}}^{\prime}=\left\{\begin{array}{c}
u_{k} \bar{u}_{k} \\
\text { or } \\
\bar{u}_{k} u_{k},
\end{array} \quad w_{J_{j}}^{\prime}=\left\{\begin{array}{c}
u_{k} \bar{u}_{k} \\
\text { or } \\
\bar{u}_{k} u_{k},
\end{array} \quad P \neq P^{\prime} .\right.\right.
$$

Each $I_{j}$ then intersects two sets of $P^{\prime}$. Call them $J_{j}$ and $J_{j+1}$.

CASE 1: Suppose $\#\left(I_{j} \cap J_{j}\right)=\#\left(I_{j} \cap J_{j+1}\right)=2^{k}$. Then if $w_{I_{j}}^{\prime}=u_{k} \bar{u}_{k}$ then $w_{J_{j}}^{\prime}=\bar{u}_{k} u_{k}$ as the overlap of the two intervals $I_{j}$ and $J_{j}$ contains precisely the word $u_{k}$, and $w_{J_{j+1}}^{\prime}=\bar{u}_{k} u_{k}$ as the overlap of $w_{I_{j}}^{\prime}$ and $w_{J_{j+1}}^{\prime}$ contains precisely the word $\bar{u}_{k}$. But then $w_{I_{j+1}}^{\prime}$ must be $u_{k} \bar{u}_{k}$. This implies $w_{I_{j}}^{\prime}=u_{k} \bar{u}_{k}$ for all $j$, so $w^{\prime}$ is a periodic point with period $2^{k+1}$, which is a contradiction as in $X$ there are no periodic points.

CASE 2: Suppose \# $\left(I_{j} \cap J_{j}\right)<\#\left(I_{j} \cap J_{j+1}\right)$, which implies \# $\left(I_{j} \cap J_{j+1}\right)$ $>2^{k}$. We show that if $w_{I_{j}}^{\prime}=u_{k} \bar{u}_{k}$ then $w_{J_{j+1}}^{\prime}=\bar{u}_{k} u_{k}$, and if $w_{I_{j}}^{\prime}=\bar{u}_{k} u_{k}$ then $w_{J_{j+1}}^{\prime}=u_{k} \bar{u}_{k}$.

Suppose $w_{I_{j}}^{\prime}=u_{k} \bar{u}_{k}$ and $w_{J_{j+1}}^{\prime}=u_{k} \bar{u}_{k}$. Let $A=w_{I_{j} \cap J_{j}}^{\prime}$ have length $\ell$, which implies the first $\ell$ symbols of $u_{k}$ are $A$. So the first $\ell$ symbols of $w_{J_{j+1}}^{\prime}$ are $A$. This implies the second $\ell$ symbols of $I_{j}$ are $A$ and as $\# A<\frac{1}{2}\left(2^{k+1}\right)$, $w_{I_{j}}^{\prime}$ begins with $A A$, but this is not all of $w_{I_{j}}^{\prime}$. The second $\ell$ symbols of $w_{J_{j+1}}^{\prime}$ must also be $A$. This says the symbol following $A A$ in $w_{I_{j}}^{\prime}$ is the first symbol of $A$, i.e. $w_{I_{j}}^{\prime}$ starts with $A A a$, and by Gottschalk and Hedlund [GH] this cannot happen in the Morse system. Hence if $w_{I_{j}}^{\prime}=u_{k} \bar{u}_{k}$ then $w_{J_{j+1}}^{\prime}=\bar{u}_{k} u_{k}$. Similarly if $w_{I_{j}}^{\prime}=\bar{u}_{k} u_{k}$ then $w_{J_{j+1}}^{\prime}=u_{k} \bar{u}_{k}$.

Now let $t=\#\left(I_{j} \cap J_{j}\right)$. Then $\sigma^{-t}\left(I_{j}\right)=J_{j+1}$ and $\sigma^{-t}\left(w_{I_{j}}^{\prime}\right)=\bar{w}_{J_{j+1}}^{\prime}$ for all $j$, so $\sigma^{-t}\left(w^{\prime}\right)=\bar{w}^{\prime}$, hence $\sigma^{-2 t}\left(w^{\prime}\right)=w^{\prime}$, and consequently $w^{\prime}$ is a periodic point with period $2 t$, which is a contradiction.

CASE 3: Suppose \# $\left(I_{j} \cap J_{j}\right)>\#\left(I_{j} \cap J_{j+1}\right)$, which implies \# $\left(I_{j} \cap J_{j+1}\right)$ $<2^{k}$. A completely symmetric argument to Case 2 holds and a contradiction arises.

Hence the partition is unique. 
Corollary 2.2. For each $w^{\prime} \in X$ there exists a unique partition of $\mathbb{Z}$ into intervals of length $2^{k}$ so that the subword of $w^{\prime}$ on each interval of this partition is either $u_{k}$ or $\bar{u}_{k}$.

Proof. Just notice that $u_{k+1}=u_{k} \bar{u}_{k}$ and $\bar{u}_{k+1}=\bar{u}_{k} u_{k}$ and so any partition into occurrences of $u_{k} \bar{u}_{k}$ or $\bar{u}_{k} u_{k}$ is a partition into occurrences of $u_{k+1}$ and $\bar{u}_{k+1}$ and vice versa.

Proposition 2.3. For $w^{\prime} \in X$, partition $\mathbb{Z}$ into intervals according to occurrences of the subwords $u_{k}$ and $\bar{u}_{k}$ as described above and let $t_{k}\left(w^{\prime}\right)$ be the position occupied by 0 in its interval, $0 \leq t_{k}\left(w^{\prime}\right)<2^{k}$. The functions $t_{k}$ are continuous.

Proof. Suppose not, that is, suppose we have a value $k$ and a sequence of points $w_{i}$ converging to some point $w^{\prime}$ but the values $t_{k}\left(w_{i}\right)$ do not converge to $t_{k}\left(w^{\prime}\right)$. Without loss of generality we can assume that $t_{k}\left(w_{i}\right)=t$ but $t \neq t_{k}\left(w^{\prime}\right)$. As $i$ increases, more and more terms of $w_{i}$ and $w^{\prime}$ agree. It follows that $w^{\prime}$ must have two partitions into subwords $u_{k}$ and $\bar{u}_{k}$, one consisting of intervals with 0 at index $t$, and one with 0 at index $t_{k}\left(w^{\prime}\right)$ in its interval. This conflicts with the uniqueness of the partition and implies the result.

Notice that $t_{k+1}\left(w^{\prime}\right)$ must be either $t_{k}\left(w^{\prime}\right)$ or $t_{k}\left(w^{\prime}\right)+2^{k}$. Let $\alpha_{k}\left(w^{\prime}\right)=$ $\left\{\alpha_{1}, \ldots, \alpha_{k}\right\}$ be the binary expansion of $t_{k}\left(w^{\prime}\right)$, i.e.

$$
t_{k}\left(w^{\prime}\right)=\sum_{i=0}^{k-1} \alpha_{i+1} 2^{i} .
$$

Then $\alpha_{k+1}\left(w^{\prime}\right)$ extends $\alpha_{k}\left(w^{\prime}\right)$, agreeing with it on its first $k$ digits. Let $\alpha\left(w^{\prime}\right)$ be the limit value in $\{0,1\}^{\mathbb{N}}$. Let $S$ be the adding machine acting on $\{0,1\}^{\mathbb{N}}$. Then it is a simple observation that $\alpha\left(\sigma\left(w^{\prime}\right)\right)=S\left(\alpha\left(w^{\prime}\right)\right)$. That is, the continuous map $\alpha$ factors the action of $\sigma$ onto that of $S$. It is also clear that $\alpha$ is not $1-1$ as $\alpha\left(\bar{w}^{\prime}\right)=\alpha\left(w^{\prime}\right)$. On the other hand, it is not difficult to see that $\alpha$ is everywhere 2-1 and that the two inverse images of any point form a pair consisting of $w^{\prime}$ and $\bar{w}^{\prime}$. This is the factor map we alluded to earlier.

We can now build the structures that Keane and Hamachi laid out to obtain a finitary orbit equivalence.

2.4. $k$-canonical cylinder sets. As we have seen, the 0th coordinate of the infinite sequence $w^{\prime}$ is at some position $0 \leq t_{k}\left(w^{\prime}\right)<2^{k}$ in one of the words $u_{k}$ or $\bar{u}_{k}$. Define

$$
\begin{aligned}
& u_{k}(i)=\left\{w^{\prime} \in X \mid t_{k}\left(w^{\prime}\right)=i \text { and } w_{0}^{\prime}=u_{k, i}\right\}, \\
& \bar{u}_{k}(i)=\left\{w^{\prime} \in X \mid t_{k}\left(w^{\prime}\right)=i \text { and } w_{0}^{\prime}=\bar{u}_{k, i}\right\},
\end{aligned}
$$

where $u_{k, i}$ (resp. $\bar{u}_{k, i}$ ) represents the $i$ th coordinate of $u_{k}$ (resp. $\bar{u}_{k}$ ) for $0 \leq i<2^{k}$. The two sets $u_{k}(i)$ and $\bar{u}_{k}(i)$ are obtained by taking the clopen 
set where $t_{k}\left(w^{\prime}\right)=i$ and splitting it according to whether the symbol at the origin is a 0 or a 1 (be careful here: which one is $u_{k}(i)$ and which is $\bar{u}_{k}(i)$ depends on the symbols in $u_{k}$ and $\bar{u}_{k}$ at index $i$ ), and hence these are clopen sets. These $2^{k+1}$ sets, all of equal measure, are called the $k$-canonical cylinders in $X$. Note that

$$
\begin{aligned}
& u_{k}(0) \stackrel{\sigma}{\rightarrow} u_{k}(1) \stackrel{\sigma}{\rightarrow} \cdots \stackrel{\sigma}{\rightarrow} u_{k}\left(2^{k}-1\right), \\
& \bar{u}_{k}(0) \stackrel{\sigma}{\rightarrow} \bar{u}_{k}(1) \stackrel{\sigma}{\rightarrow} \cdots \stackrel{\sigma}{\rightarrow} \bar{u}_{k}\left(2^{k}-1\right) .
\end{aligned}
$$

A finite or countable union of canonical cylinders is called a cylinder in $X$.

The canonical cylinders do not quite separate points in $X$. To see this, notice that if $w^{\prime} \in u_{k}(i)$ then we know the symbolic name of $w^{\prime}$ for indices $-i,-i+1, \ldots,-i+2^{k}-1$, and so long as both $t_{k}\left(w^{\prime}\right)$ and $2^{k}-t_{k}\left(w^{\prime}\right)$ go to infinity, knowing the sets $u_{k}(i)$ containing $w^{\prime}$ will determine $w^{\prime}$ uniquely. This covers all but four orbits in $X$. If we let $m$ be $w$ reflected, i.e. $m_{-i}=w_{1-i}$ for $i \geq 1$, then we can give four unusual words in $X, m w, \bar{m} w, m \bar{w}, \overline{m w}$. It is precisely on the orbits of these four words that one of $t_{k}\left(w^{\prime}\right)$ or $2^{k}-t_{k}\left(w^{\prime}\right)$ remains bounded. From a finitary perspective this is not an issue as these four orbits are a set of measure zero.

2.5. Cylinder partition. A cylinder partition of $X$ is a set $\mathcal{C}$ of pairwise disjoint cylinders such that

$$
\sum_{c \in \mathcal{C}} \mu(c)=1
$$

2.6. Canonical cylinder partition. The canonical cylinders $\left\{u_{k}(i), \bar{u}_{k}(i) \mid\right.$ $\left.0 \leq i<2^{k}\right\}$ form a cylinder partition of $X$, called the $k$ th canonical cylinder partition of $X$.

2.7. Tower partitions. A tower partition is a cylinder partition $\mathcal{C}$ endowed with an equivalence relation in which each equivalence class consists of canonical cylinders all either of the form $u_{k}(i)$ where $k$ is fixed but $i$ varies, or of the form $\bar{u}_{k}(i)$ where again $k$ is fixed but $i$ varies, and in which the elements of each equivalence class are ordered by indexing them with some initial interval in $\mathbb{N}$. That is, a column will look like $\left\{u_{k}\left(i_{0}\right), u_{k}\left(i_{1}\right), \ldots, u_{k}\left(i_{h-1}\right)\right\}$, or a similar list of $\bar{u}_{k}\left(i_{j}\right)$ where these cylinders are ordered by the subscript on $i$. What this ordering does is give each element of a column except the first one a follower element in the column and each element except the last one a predecessor element in the column.

Each ordered equivalence class $C$ of the tower partition $\mathcal{C}$ is called a tower or a column of $\mathcal{C}$. The number of elements in $C$ is called the height of the tower $C$. 
By the support of $C$ we mean

$$
\operatorname{Supp}(C)=\bigcup_{c \in C} c
$$

2.8. Canonical tower partition $\mathcal{P}_{k}(k \geq 0)$. Each canonical cylinder partition $\left\{u_{k}(i), \bar{u}_{k}(i) \mid 0 \leq i<2^{k}\right\}(k \geq 0)$ consisting of two equivalence classes $\left\{u_{k}(i) \mid 0 \leq i<2^{k}\right\}$ and $\left\{\bar{u}_{k}(i) \mid 0 \leq i<2^{k}\right\}$ ordered by the index $i$ is called the $k$ th canonical tower partition of $X$ and is denoted by $\mathcal{P}_{k}$. Notice that the order on the columns is simply the order induced by the action of $\sigma$.

2.9. Common extension. Let $C=\left\{c_{0}, \sigma^{n_{1}} c_{0}, \sigma^{n_{2}} c_{0}, \ldots, \sigma^{n_{k}} c_{0}\right\}$ be an ordered column of a tower partition $\mathcal{C}$ and $c_{0}^{\prime} \subseteq c_{0}$ be a canonical cylinder. Then we call the ordered set $\left\{c_{0}^{\prime}, \sigma^{n_{1}} c_{0}^{\prime}, \sigma^{n_{2}} c_{0}^{\prime}, \ldots, \sigma^{n_{k}} c_{0}^{\prime}\right\}$ a common extension of $C$ by $c_{0}^{\prime}$.

A tower partition $\hat{\mathcal{C}}$ is said to be a common extension of a tower partition $\mathcal{C}$ if each column of $\hat{\mathcal{C}}$ is a common extension of a column of $\mathcal{C}$.

2.10. Tower refinement. Let $\mathcal{C}$ be a tower partition and $\hat{\mathcal{C}}$ be a common extension of $\mathcal{C}$. Then a tower partition $\mathcal{C}^{\prime}$ is a tower refinement of $\mathcal{C}$ if each column of $\mathcal{C}^{\prime}$ is a union of columns of $\widehat{\mathcal{C}}$ and the order on $\mathcal{C}^{\prime}$ extends the order on $\widehat{\mathcal{C}}$ in that it preserves the follower and predecessor relations where they are defined and extends them by concatenating the columns in some order. Note that if $\mathcal{C}^{\prime}$ is a tower refinement of $\mathcal{C}$, then each $c^{\prime} \in \mathcal{C}^{\prime}$ is contained in a unique $c \in \mathcal{C}$, yielding a map $\pi: \mathcal{C}^{\prime} \rightarrow \mathcal{C}$ which preserves the measure $\mu$ in that the measure of the support of the pull back of a set in $\mathcal{C}$ is the same as its measure.

REMARK. The canonical tower partition $\mathcal{P}_{k+1}$ is a tower refinement of the canonical tower partition $\mathcal{P}_{k}$.

For all $l, k \in \mathbb{N}$ the words $u_{l+k}$ and $\bar{u}_{l+k}$ are concatenations of $u_{l}$ and $\bar{u}_{l}$ and we can make the following observation.

LEMMA 2.11. In $u_{l+k}=a_{1} \cdots a_{2^{k}}$, where $\ell\left(a_{i}\right)=2^{l}$ and $a_{i}=u_{l}$ or $\bar{u}_{l}$, each pair $\left(a_{2 j-1}, a_{2 j}\right)$ is either $u_{l+1}$ or $\bar{u}_{l+1}$.

We know $u_{0}=0, \bar{u}_{0}=1, u_{k+1}=u_{k} \bar{u}_{k}, \bar{u}_{k+1}=\bar{u}_{k} u_{k}$ and for $k \geq 0$, all $u_{k}=u_{k, 1} \cdots u_{k, 2^{k}}$ have length $2^{k}$, half of symbols are 0 and half are 1 . Let $S\left(2^{k}\right)$ be the symmetric group on $\left\{1, \ldots, 2^{k}\right\}$ and $G_{k}=\left\{\varrho \in S\left(2^{k}\right) \mid\right.$ $\left.u_{k, i}=\bar{u}_{k, \varrho(i)}\right\}$. Then $\# G_{k}=\left(2^{k-1} !\right)^{2}$. Said more plainly, $G_{k}$ is the collection of all permutations of $\left\{1, \ldots, 2^{k}\right\}$ that rearrange $u_{k}$ into $\bar{u}_{k}$. Notice that by symmetry its elements also rearrange $\bar{u}_{k}$ into $u_{k}$. Our next goal, and the central construction needed for the proof, is to show how to construct elements of $G_{k}$ that are monotone on a large part of their domain. This construction, a "nesting argument", is a method that goes back to [ORW]. 
Set

$$
\begin{aligned}
m(\varrho) & =\frac{1}{2^{k}} \max \left\{\# S: S \subseteq\left\{1, \ldots, 2^{k}\right\} \text { and } \varrho \text { preserves order on } S\right\}, \\
D_{k} & =\max \left\{m(\varrho) \mid \varrho \in G_{k}\right\} .
\end{aligned}
$$

Proposition 2.12. $\lim _{k \rightarrow \infty} D_{k}=1$.

Proof. First we show $D_{k+1} \geq D_{k}$. To see this just note that $u_{k+1}=u_{k} \bar{u}_{k}$ and $\bar{u}_{k+1}=\bar{u}_{k} u_{k}$. So if $\varrho \in G_{k}$ then $\varrho^{\prime}$, the concatenation of $\varrho$ with $\varrho^{-1}$ (or $\varrho$ for that matter) will be in $G_{k+1}$ and $m\left(\varrho^{\prime}\right) \geq m(\varrho)$. Thus it is enough to show this convergence to 1 along a subsequence.

We will now give an inductive "nesting" procedure that constructs a map $\varrho$ in the symmetric group of $\left\{1, \ldots, 2^{k_{1}+\cdots k_{N}}\right\}$. Let $k_{1}, \ldots, k_{N} \in \mathbb{N}$ and $l_{1}=k_{1}+\cdots+k_{N-1}$. Then $u_{k_{1}+\cdots+k_{N}}=u_{l_{1}+k_{N}}=a_{1}^{l_{1}} \cdots a_{2^{k_{N}}}^{l_{1}}$. By Lemma 2.11 we have $\left(a_{2 j-1}^{l_{1}}, a_{2 j}^{l_{1}}\right)=\left(u_{l_{1}}, \bar{u}_{l_{1}}\right)$ or $\left(\bar{u}_{l_{1}}, u_{l_{1}}\right)$. Let $\left(a_{2 j-1}^{l_{1}}, a_{2 j}^{l_{1}}\right)=$ $\left(u_{l_{1}}, \bar{u}_{l_{1}}\right)$ and so $\left(\bar{a}_{2 j-1}^{l_{1}}, \bar{a}_{2 j}^{l_{1}}\right)=\left(\bar{u}_{l_{1}}, u_{l_{1}}\right)$. Let us now define a mapping $\varrho_{l_{1}, k_{N}}$ on $\bigcup_{j=1}^{2^{k_{N}-1}}\left[2(j-1) 2^{l_{1}}+1, \ldots, 2 j 2^{l_{1}}\right]$ by $\varrho_{l_{1}, k_{N}}(t)=t+2^{l_{1}}(t>0)$. Then $\varrho_{l_{1}, k_{N}}$ takes $a_{2 j-1}^{l_{1}}$ to $\bar{a}_{2 j}^{l_{1}}$ as a positive translation. Since $a_{2 j-1}^{l_{1}}=\bar{a}_{2 j}^{l_{1}}$, we say $\varrho_{l_{1}, k_{N}}$ matches $a_{2 j-1}^{l_{1}}$ to $\bar{a}_{2 j}^{l_{1}}$. Also $\varrho_{l_{1}, k_{N}}$ matches $a_{2 j}^{l_{1}}$ to $\bar{a}_{2 j+1}^{l_{1}}$ if it happens that $a_{2 j}^{l_{1}}=\bar{a}_{2 j+1}^{l_{1}}$. Clearly $\varrho_{l_{1}, k_{N}}$ is monotone on the indices where it is defined, and it is not defined on the last $2^{l_{1}}=2^{k_{1}+\cdots+k_{N-1}}$ symbols.

In the second stage we define $\varrho_{l_{2}, k_{N-1}}$, where $l_{2}=k_{1}+\cdots+k_{N-2}$, on the remaining indices in $\left\{1, \ldots, 2^{l_{1}+k_{N}}\right\}$ corresponding to the subwords $a_{2 j}^{l_{1}}$ which were not matched by $\varrho_{l_{1}, k_{N}}$, i.e. if $a_{2 j}^{l_{1}}$ and $\bar{a}_{2 j+1}^{l_{1}}$ are flips of each other. Let $a_{2 j}^{l_{1}}$ and $\bar{a}_{2 j+1}^{l_{1}}$ be flips of each other for some $j$. Then $a_{2 j}^{l_{1}}=u_{l_{1}}$ or $\bar{u}_{l_{1}}$. Let $a_{2 j}^{l_{1}}=u_{l_{1}}$ and then $\bar{a}_{2 j+1}^{l_{1}}=\bar{u}_{l_{1}}$.

Write $a_{2 j}^{l_{1}}=u_{l_{1}}=a_{1}^{l_{2}} \cdots a_{2^{k_{N-1}}}^{l_{2}}$, and hence $\bar{a}_{2 j+1}^{l_{1}}=\bar{a}_{1}^{l_{2}} \cdots \bar{a}_{2^{k_{N-1}}}^{l_{2}}$. Now define a mapping $\varrho_{l_{2}, k_{N-1}}$ on $\bigcup_{j=1}^{2^{k_{N-1}-1}}\left[2(j-1) 2^{l_{2}}+1, \ldots, 2 j 2^{l_{2}}\right]$ by $\varrho_{l_{2}, k_{N-1}}(t)$ $=t+2^{l_{2}}(t>0)$. Then $\varrho_{l_{2}, k_{N-1}}$ matches $a_{2 j-1}^{l_{2}}$ to $\bar{a}_{2 j}^{l_{2}}$ and matches $a_{2 j}^{l_{2}}$ to $\bar{a}_{2 j+1}^{l_{2}}$ if $a_{2 j}^{l_{2}}=\bar{a}_{2 j+1}^{l_{2}}$, and is monotone where it is defined. Now construct $\varrho_{l_{3}, k_{N-2}}$ on the remaining symbols corresponding to the subwords $a_{2 j}^{l_{2}}$ which were not matched by $\varrho_{l_{2}, k_{N-1}}$. Proceeding inductively in this way through $N$ steps we construct the maps $\varrho_{l_{4}, k_{N-3}}, \varrho_{l_{5}, k_{N-4}}, \ldots, \varrho_{l_{N}, k_{1}}$.

Now let $\varrho=\varrho_{l_{1}, k_{N}} \cup \varrho_{l_{2}, k_{N-1}} \cup \cdots \cup \varrho_{l_{N}, k_{1}}$. Then

$$
\begin{aligned}
\operatorname{Dom}(\varrho) & =\operatorname{Dom}\left(\varrho_{l_{1}, k_{N}}\right) \cup \operatorname{Dom}\left(\varrho_{l_{2}, k_{N-1}}\right) \cup \cdots \cup \operatorname{Dom}\left(\varrho_{l_{N}, k_{1}}\right) \\
& \subset\left\{1, \ldots, 2^{k_{1}+\cdots+k_{N}}\right\} .
\end{aligned}
$$

Clearly $\varrho$ is monotone where it is defined. 
We now estimate the number of symbols on which $\varrho$ is not defined. Suppose at the first stage we remove $z_{1}$ symbols on which $\varrho$ is not defined and match on $M_{1} \geq \frac{1}{2}\left(L-z_{1}\right)$ symbols where $L=2^{k_{1}+\cdots+k_{N}}$. At the second stage from the remaining $L-M_{1}-z_{1}$ symbols we remove $z_{2}$ symbols and match on $M_{2} \geq \frac{1}{2}\left(L-M_{1}-z_{1}-z_{2}\right)$ symbols. At the $k$ th stage we remove $z_{k}$ symbols and match on $M_{k} \geq \frac{1}{2}\left(L-\left(M_{1}+\cdots+M_{k-1}\right)-\left(z_{1}+\cdots+z_{k}\right)\right)$ symbols. Hence the number of symbols where $\varrho$ is not defined is $\leq \sum_{i=1}^{N} z_{i}+L / 2^{N}$.

We back up now and set some parameters. For $\varepsilon>0$, choose $N$ so that $1 / 2^{N}<\varepsilon / 2$. For $N$ and $\varepsilon$ now fixed, choose $k$ so that $N / 2^{k}<\varepsilon / 2$. Now let $k_{1}=\cdots=k_{N}=k$. We have

$$
\begin{gathered}
z_{1}=2^{k_{1}+\cdots+k_{N-1}}=2^{(N-1) k}=\frac{L}{2^{k}}, \quad z_{2} \leq \frac{L}{2^{k}}, \quad z_{3} \leq \frac{L}{2^{k}} \quad \text { and so on, } \\
\sum_{i=1}^{N} z_{i} \leq \frac{N L}{2^{k}}<\frac{\varepsilon L}{2} .
\end{gathered}
$$

Hence $\varrho$ is defined and monotone on a subset of size $\geq(1-\varepsilon) L$, and $u_{L, \varrho^{-1}(i)}$ $=\bar{u}_{L, i}$ where defined.

Since the numbers of 0 's and 1's in $u_{L}$ and $\bar{u}_{L}$ are identical, we can extend $\varrho$ to all of $\{1, \ldots, L\}$ with $\bar{u}_{L, i}=u_{L, \varrho^{-1}(i)}$, i.e. $\varrho \in G_{\log _{2}(L)}$ and $m(\varrho) \geq(1-\varepsilon) L / L=1-\varepsilon$ and hence

$$
1 \geq D_{\log _{2}(L)} \geq 1-\varepsilon \quad \text { where } \quad L=2^{N k} .
$$

We know $G_{k}=\left\{\varrho \in S\left(2^{k}\right) \mid \varrho\right.$ carries $u_{k}$ to $\left.\bar{u}_{k}\right\}$. If $k>k_{0}$ then $u_{k}$ and $\bar{u}_{k}$ split into $2^{k-k_{0}}$ subwords, each of length $2^{k_{0}}$ and each either $u_{k_{0}}$ or $\bar{u}_{k_{0}}$.

Define

$$
G_{k, k_{0}}=\left\{\varrho \in G_{k} \mid \varrho \text { acts as a translation on }\left[(i-1) 2^{k_{0}}+1, \ldots, i 2^{k_{0}}\right]\right\} \text {. }
$$

By this we mean that for each $i$ there exists $t_{i}\left(t_{i}=s_{i} 2^{k_{0}}\right.$ for some $\left.s_{i} \in \mathbb{Z}\right)$ such that if $j \in\left\{(i-1) 2^{k_{0}}+1, \ldots, i 2^{k_{0}}\right\}$ then $\varrho(j)=j+t_{i}$.

Define

$$
D_{k, k_{0}}=\max \left\{m(\varrho) \mid \varrho \in G_{k, k_{0}}\right\} .
$$

Clearly $D_{k, k_{0}} \leq D_{k}$ as $G_{k, k_{0}} \subseteq G_{k}$.

Proposition 2.13. For all $k_{0} \in \mathbb{N}, \lim _{k \rightarrow \infty} D_{k, k_{0}}=1$.

Proof. For $k_{0}<k$, write $u_{k}$ as a concatenation of occurrences of $u_{k_{0}}$ and $\bar{u}_{k_{0}}$. If one replaces each occurrence of $u_{k_{0}}$ by a 0 and each occurrence of $\bar{u}_{k_{0}}$ by a 1 one obtains the word $u_{k-k_{0}}$. (This is just moving backward through the substitution.) For any $\varrho \in G_{k-k_{0}}$ we can define a $\widehat{\varrho} \in G_{k, k_{0}}$ as follows. For $j \in\left\{(i-1) 2^{k_{0}}+1, \ldots, i 2^{k_{0}}\right\}$ set

$$
\widehat{\varrho}(j)=2^{k_{0}}(\varrho(i)-1)+\left(j-(i-1) 2^{k_{0}}\right) .
$$


Thus $\widehat{\varrho}$ permutes consecutive blocks of length $2^{k_{0}}$ in $\left\{1, \ldots, 2^{k}\right\}$ exactly as $\varrho$ permutes elements of $\left\{1, \ldots, 2^{k-k_{0}}\right\}$. It follows that $\varrho \in G_{k, k_{0}}$ and $m(\widehat{\varrho})=m(\varrho)$, and the result follows.

3. Binary odometer. In this section we discuss the basic definitions in the case of binary odometer. For details see [HK], [R1].

3.1. Binary odometer and the canonical cylinders. The binary odometer or adding machine is well known but for completeness we give a brief description. Let $Y=\{0,1\}^{\mathbb{N}}$. Then $Y$ is a product topological space arising from the discrete topology on each of its coordinate space $\{0,1\}$. It is metrizable. The metric

$$
d\left(y, y^{\prime}\right)=2^{-l} \quad \text { where } \quad l=\min \left\{|i|: y_{i} \neq y_{i}^{\prime}\right\}
$$

generates the product topology on $Y$. The open balls in the product topology are

$$
B\left(y, 2^{-l}\right)=\left\{y^{\prime}=\left(y_{n}^{\prime}\right) \in Y: y_{i}=y_{i}^{\prime}, 0 \leq i \leq l\right\}
$$

Let

$$
W=\{0,1\}^{*}=\bigcup_{l \in \mathbb{N}}\{0,1\}^{l}
$$

denote the set of all binary words. If $d \in W$ then $|d|$ denotes its length; the empty word $\emptyset$ has length 0 . Each word $d \in W$ gives rise to the subset

$$
\{y \in Y \mid y \text { begins with } d\}
$$

of $Y$, which we call a cylinder in $Y$ and also denote by the same letter $d$. If $y \in d$ then by (2) we have

$$
d=B\left(y, 2^{-|d|}\right) .
$$

The clopen sets of $Y$ are precisely the finite unions of cylinders. We now set

$\mathfrak{B}:=$ the Borel $\sigma$-algebra of $Y$ generated by the cylinders in $Y$,

$$
\nu:=(1 / 2,1 / 2)^{\mathbb{N}}=\text { the product measure on }(Y, \mathfrak{B}) .
$$

We define $S$ acting on $Y$ by addition with carry to the right. This map acts as a cyclic permutation on the $2^{k}$ cylinders of length $k$. This shows $S$ is a homeomorphism and that it preserves the measure $\nu$. We refer to such a cylinder as a dyadic interval, because if we consider a sequence in $Y$ to be the binary expansion of a point in the unit interval, these sets correspond to dyadic intervals.

3.2. Cylinder partition etc. The definitions of the cylinder partition, tower partition, common extension, tower refinement in the case of the binary odometer are exactly the same as for the Morse sequence, but in this 
case by the canonical tower partition $\mathcal{D}_{k}$ we mean the tower partition of height $2^{k}$ consisting of all the $k$-canonical cylinders endowed with an increasing order up the tower as induced by the action of $S$.

\section{Construction of the almost continuous Kakutani equivalence.}

We follow the Keane-Hamachi format here, constructing two sequences of towers with a web of commuting tower maps between them. By Proposition 2.1, there exists a sequence $\left\{k_{n}\right\}_{n \geq 0}$ with $0=k_{0}<k_{1}<\cdots$ such that

$$
D_{k_{n+1}, k_{n}}>1-\frac{1}{10 \cdot 2^{n}} \quad(n \geq 0) .
$$

Let $\left\{\mathcal{P}_{k_{n}}\right\}$ be the corresponding sequence of the canonical tower partitions of $X$ and $\left\{\mathcal{D}_{k_{n}}\right\}$ be that of $Y$.

We first define tower maps $\phi_{i}$ that carry two levels in each $\mathcal{P}_{k_{i}}$ to each interval in $\mathcal{D}_{k_{i}}$, one chosen from each column. Denote the two columns of $\mathcal{P}_{k_{i}}$ by $C_{i, 0}$ which has base $J_{k_{i}}^{0,0}$ and $C_{i, 1}$ which has base $J_{k_{i}}^{0,1}$. Then $\phi_{i}$ is completely defined by giving two permutations $\eta_{i, 0}, \eta_{i, 1}$ of $\left\{1, \ldots, 2^{k_{i}}\right\}$, i.e. $\eta_{i, 0}, \eta_{i, 1} \in S\left(2^{k_{i}}\right)$ that describe how the levels of each of these columns are reordered to be paired up with a single interval in $\mathcal{D}_{k_{i}}$.

Level $j$ in $C_{i, 0}$ is mapped by $\phi_{i}$ to level $\eta_{i, 0}(j)$ in the single column of $\mathcal{D}_{k_{i}}$, and level $j$ in $C_{i, 1}$ is mapped to level $\eta_{i, 1}(j)$ in this single column. Select elements $\varrho_{n+1} \in G_{k_{n+1}, k_{n}}$ for $n \geq 0$ that achieve $D_{k_{n+1}, k_{n}}$. Let $\eta_{1,0}=$ Id and $\eta_{1,1}=\eta_{1,0} \varrho_{1}^{-1}=\varrho_{1}^{-1}$.

Suppose $\eta_{i, 0}$ and $\eta_{i, 1}$ are defined. Take a level $j$ in $C_{i+1,0}$ and let $j^{\prime}=$ $(j-1)\left(\bmod 2^{k_{i}}\right)+1$. Then $j^{\prime} \in\left\{1, \ldots, 2^{k_{i}}\right\}$ and level $j$ in $\mathcal{P}_{k_{i+1}}$ is either in a common extension of $C_{i, 0}$ or of $C_{i, 1}$. If it is in $C_{i, t}(t=0,1)$ then $\eta_{i+1,0}(j)=\eta_{i, t}\left(j^{\prime}\right)+j^{\prime \prime} 2^{k_{i}}$ where $j^{\prime \prime}=\left\lfloor(j-1) / 2^{k_{i}}\right\rfloor$. That is, $\eta_{i+1,0}$ is the concatenation of copies of $\eta_{i, 0}$ and $\eta_{i, 1}$ in the order prescribed by the occurrences of extensions of $C_{i, 0}$ and $C_{i, 1}$ in $C_{i+1,0}$. Having defined $\eta_{i+1,0}$ we set

$$
\eta_{i+1,1}=\eta_{i+1,0} \varrho_{i+1}^{-1} .
$$

Now we define maps $\psi_{i+1}$ which carry each level in $\mathcal{D}_{k_{i+1}}$ to a level of a column in $\mathcal{P}_{k_{i}}$. Suppose we have a level at height $j$ in $\mathcal{D}_{k_{i+1}}$. Then $\phi_{i+1}^{-1}$ of this level consists of two levels, one in each column of $\mathcal{P}_{k_{i+1}}$; call them $L_{0}(j)$ and $L_{1}(j)$. Since $\varrho_{i+1}$ works as a translation on intervals of length $2^{k_{i}}$, both $L_{0}(j)$ and $L_{1}(j)$ come from the same level of the same column of $\mathcal{P}_{k_{i}}$, i.e. $\pi\left(L_{0}(j)\right)=\pi\left(L_{1}(j)\right) \in \mathcal{P}_{k_{i}}$. Define

$$
\psi_{i+1}(j)=\pi\left(L_{0}(j)\right)=\pi\left(L_{1}(j)\right) .
$$

The permutations $\eta_{i, 0}$ and $\eta_{i, 1}$ are defined as they are so as to make the following result hold. 
Proposition 4.1. The following diagram of tower maps commutes:

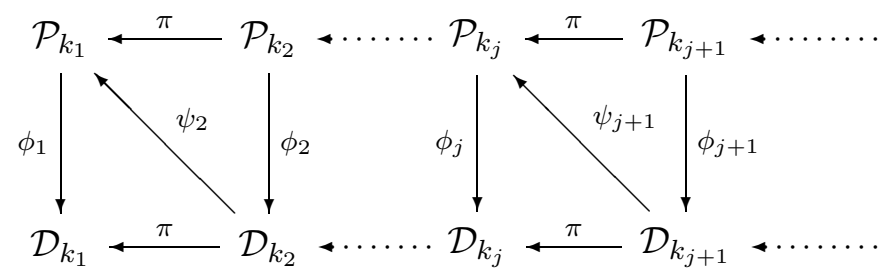

We now show that the inverse limits through these tower maps converge to the finitary Kakutani equivalence we seek. Consider a nested sequence of sets $c_{n} \in \mathcal{P}_{k_{n}}$, i.e. $c_{n+1} \subseteq c_{n}$. The diagram implies that the sets $\phi_{n}\left(c_{n}\right) \in \mathcal{D}_{k_{n}}$ also form a nested sequence of sets. Symmetrically, if $d_{n} \in \mathcal{D}_{k_{n}}$ are nested, then so will be their images $\psi_{n}\left(d_{n}\right) \in \mathcal{P}_{k_{n-1}}$. In both $X$ and $Y$ we can define equivalence relations where two points are equivalent iff they always lie in the same element of $\mathcal{P}_{k_{n}}$ or $\mathcal{D}_{k_{n}}$. The inverse limit of the maps $\phi_{n}$ will be a map $\phi$ from the classes in $X$ to those in $Y$. The inverse limit of the $\psi_{n}$ will be $\phi^{-1}$. In $Y$ all equivalence classes consist of single points. Not so in $X$. As described earlier, $X$ possesses four rather special orbits given by placing the origin at an index in one of the four words $m w, \bar{m} w, m \bar{w}$ and $\bar{m} \bar{w}$. Suppose $x$ is such a point where the origin sits at a position $l$ symbols into the $m$ or $\bar{m}$ half of the word. This is equivalent to saying that for all $n$ large enough, the $x$ lies $l$ levels from the top of the tower $\mathcal{P}_{n}$. In this case the equivalence class consists of two points, the other one being obtained by switching $w$ and $\bar{w}$ on the second half of the word, leaving the origin where it sits. Symmetrically, if $x$ is such a point but with the origin at a position $l$ symbols into the $w$ or $\bar{w}$ portion of the word. This is equivalent to saying that for all $n$ large enough, $x$ lies $l$ levels from the bottom of the tower $\mathcal{P}_{n}$. Again the equivalence class consists of two points, the other one being obtained by switching $m$ and $\bar{m}$ on the first half of the word.

We are going to remove from both $X$ and $Y$ subsets of measure zero consisting of those points which always remain within some bounded distance of the top or bottom of the towers. In both $X$ and $Y$ these are countable subsets and consist of full equivalence classes. Call them $N$ and $N^{\prime}$ and now define

$$
N_{X}=N \cup \phi^{-1}\left(N^{\prime}\right) \quad \text { and } \quad N_{Y}=\phi\left(N_{X}\right)=\phi(N) \cup N^{\prime} .
$$

Both $N_{X}$ and $N_{Y}$ are countable sets and hence of measure zero. In $X-N_{X}$ every nested chain of sets $c_{n}$ intersects to a single point. For $x \in X$ if we let $c_{n}(x)$ be that element of $\mathcal{P}_{k_{n}}$ that contains $x$, then we have

$$
\bigcap_{n} c_{n}(x)=\{x\} \quad \text { for all } x \in X-N_{X} \text {. }
$$


Similarly, if $d_{n}(y)$ is that element of $\mathcal{D}_{k_{n}}$ containing $y$, then

$$
\bigcap_{n} d_{n}(y)=\{y\} \quad \text { for all } y \in Y .
$$

The maps $\phi$ and $\psi$ were defined on equivalence classes and so as maps on $X-N_{X}$ and $Y-N_{Y}$ are carrying singleton sets to singleton sets. Let $\Phi: X-N_{X} \rightarrow Y-N_{Y}$ and $\Psi=\Phi^{-1}: Y-N_{Y} \rightarrow X-N_{X}$ be the corresponding point maps, i.e. $\phi(\{x\})=\{\Phi(x)\}$ etc.

Lemma 4.2. Both $\Phi$ and $\Psi$ are continuous and hence $\Phi$ is an almost continuous isomorphism of the two measure spaces.

Proof. Notice that for $x \in X-N_{X}, \Phi\left(c_{n}(x)\right) \subseteq d_{n}(\Phi(x))$, and for $y \in$ $Y-N_{Y}, \Psi\left(d_{n+1}(y)\right) \subseteq c_{n}(\Psi(y))$. All the sets $c_{n}(x)$ and $d_{n}(y)$ are clopen. Along any chain that intersects to a single point, the diameters of the sets in the chain must decrease to 0 . Continuity of $\Phi$ and $\Psi$ follows.

Proposition 4.3. $\Phi$ is an orbit equivalence from $\sigma$ to $S$.

Proof. First we show $\Phi$ carries orbits into orbits. Suppose $x$ and $\widetilde{x}$ are two points in $X-N_{X}$ and on the same orbit. Assume $\widetilde{x}=\sigma^{l}(x)$. As $x \in X-N_{X}$, once $n$ is large enough, the cylinder $c_{n}(x)$ in $\mathcal{P}_{k_{n}}$ containing $x$ will lie more than $l$ levels from both the top and bottom of the column. Let $n_{0}$ be the smallest choice for such an index $n$. It follows that $c_{n}(\widetilde{x})=\sigma^{l}\left(c_{n}(x)\right)$ for all $n \geq n_{0}$. Suppose $c_{n}(x)$ is the level at height $j_{n}(x)$ in column $t_{n} \in\{0,1\}$ in $\mathcal{P}_{k_{n}}$ and so $c_{n}(\widetilde{x})$ will be level $j_{n}(x)+l$ also in the same column $t_{n}$. The construction of $\Phi$ tells us that for $n \geq n_{0}$,

$$
d_{n}(\Phi(\widetilde{x}))=S^{\eta_{n, t_{n}}\left(j_{n}+l\right)-\eta_{n, t_{n}}\left(j_{n}\right)}\left(d_{n}(\Phi(x))\right) .
$$

As the permutations $\varrho_{n}$ act by translation on subblocks of integers of length $2^{k_{n-1}}$, the values $\eta_{n, t_{n}}\left(j_{n}+l\right)-\eta_{n, t_{n}}\left(j_{n}\right)=l^{\prime}$ are constant for all $n \geq n_{0}$ and $d_{n}(\Phi(\widetilde{x}))=S^{l^{\prime}}\left(d_{n}(\Phi(x))\right)$ for all $n \geq n_{0}$. Intersecting over $n$ we obtain $\Phi(\widetilde{x})=S^{l^{\prime}}(\Phi(x))$ and these two points are on the same $S$-orbit.

We now show that so long as $y \notin N^{\prime}, \Phi$ carries orbits onto orbits. For each $x \in X-N_{X}$, suppose $c_{n}(x)$ is at height $1 \leq j_{n}(x) \leq 2^{k_{n}}$ in its column in $\mathcal{P}_{k_{n}}$. Let $s_{n}(x)=\left\{\sigma^{k}(x) \mid-j_{n}(x)+1 \leq k \leq-j_{n}(x)+2^{k_{n}}\right\}$. This is that part of the $\sigma$-orbit of $x$ preceding and following $x$ in one transit through the tower. Similarly we can construct such transits $s_{n}^{\prime}(y)$ of a point $y$ through the towers $\mathcal{D}_{k_{n}}$. The proof that $\Phi$ carries a $\sigma$-orbit into an $S$-orbit tells us a bit more. The form of the construction of $\Phi$ gives

$$
\Phi\left(s_{n}(x)\right)=s_{n}^{\prime}(\Phi(x)) .
$$

Our proof that $\Phi$ carries an orbit into an orbit rested on the fact that for $x \notin N$ the sets $s_{n}(x)$ increase to the full orbit of $x$. But for $y \notin N^{\prime}$ also $s_{n}^{\prime}(y)$ increase to the full orbit of $y$ and hence for $x \notin N_{X}, s_{n}^{\prime}(\Phi(x))$ increase to the full orbit of $\Phi(x)$, so that $\Phi$ carries an orbit onto an orbit. 
Proposition 4.4. $\Phi$ is order preserving on a set of positive measure and hence is an almost continous Kakutani equivalence.

Proof. The permutation $\varrho_{n}$ was chosen so that $D_{k_{n}, k_{n-1}}$ was achieved on $\varrho_{n}$, so $\varrho_{n}$ preserves order on all but $1 /\left(10 \cdot 2^{n}\right)$ of its domain. Define $G_{n}$ to consist of the union of all the levels in column 1 of $\mathcal{P}_{k_{n}}$ combined with the union of all the levels in column 2 that correspond to indices on which $\varrho_{n}$ preserves order. Then $\mu\left(G_{n}\right)>1-1 /\left(10 \cdot 2^{n}\right)$. Let $G=\bigcap_{n=1}^{\infty} G_{n}$. Then

$$
\mu\left(G^{c}\right)=\mu\left(\bigcup_{n=1}^{\infty} G_{n}^{c}\right) \leq \sum_{n=1}^{\infty} \mu\left(G_{n}^{c}\right)<\sum_{n=1}^{\infty} \frac{1}{10 \cdot 2^{n}}=\frac{1}{10}, \quad \text { so } \quad \mu(G)>\frac{9}{10} .
$$

Suppose $x, \widetilde{x} \in G \cap\left(X-N_{X}\right)$ where $\widetilde{x}=\sigma^{t} x$ for some $t>0$. Once $n$ is large enough, as we are outside $N_{X}$, the points $x$ and $\widetilde{x}$ will lie in two levels $c_{n}(x)$ and $c_{n}(\widetilde{x})=\sigma^{t}\left(c_{n}(x)\right)$ in the same column of $\mathcal{P}_{k_{n}}$. Let $n$ be the least value where this happens. This means $x$ and $\widetilde{x}$ are in distinct blocks of levels of length $2^{k_{n-1}}$ in $\mathcal{P}_{k_{n}}$, but for this and all successive towers $\mathcal{P}_{m}$ with $m>k_{n}$, they are always in the same subblock of intervals of length $2^{m-1}$. In the structure of $\phi_{n}$ all rearrangements of levels that come from steps previous to step $n$ fix the blocks of length $2^{k_{n-1}}$ and hence cannot move $c_{n}(x)$ above $c_{n}(\widetilde{x})$. At step $n$, if the two levels are in the first column then $\phi_{n}$ is simply this inherited map. If they are in the second column the levels are further rearranged by the map $\varrho_{n}^{-1}$ which moves each block of $2^{k_{n-1}}$ levels as a rigid unit, and as $x, \widetilde{x} \in G_{n}$, their order is not changed. From all successive steps, as these points will lie in the same subblock, their relative positions will no longer be changed. Hence on the set $G, \Phi$ is order preserving on orbits.

This completes the proof of our main theorem. We end with two questions. The Morse system is one of the most elementary minimal substitution systems. Can this argument be generalized to show that all minimal substitution systems are almost continuously Kakutani equivalent to an adding machine? We suspect the answer here is yes. As a second and much more difficult question, all results to date in the study of almost continuous Kakutani equivalence have given positive results. That is, one demonstrates that two systems known to be measurably Kakutani equivalent are in fact almost continuously Kakutani equivalent. Is this a general theorem or are there natural examples where a measurable Kakutani equivalence cannot be obtained almost continuously? A recent preprint of del Junco and Sahin [JS] shows that for orbit equivalence there are no such examples. Very recent and as yet unwritten work of del Junco, Rudolph and Weiss would prove that indeed if two systems are measurably Kakutani equivalent, then they are almost continuously Kakutani equivalent in the sense defined here. If this is correct it indicates the definition of almost continuous Kakutani equivalence should 
be strengthened to require the subsets on which the induced maps are conjugate to be almost open. This stronger definition is used and verified in [RR1] for Markov chains. It is an open question if this can be done for the Morse system and the binary odometer, the systems considered here.

\section{References}

[CKL] E. M. Coven, M. S. Keane and M. Lemasurier, A characterization of the Morse minimal set up to topological conjugacy, Ergodic Theory Dynam. Systems, to appear.

[D] H. Dye, On groups of measure preserving transformations I, Amer. J. Math. 81 (1959), 119-159.

[GH] W. H. Gottschalk and G. A. Hedlund, A characterization of the Morse minimal set, Proc. Amer. Math. Soc. 15 (1964), 70-74.

[HK] T. Hamachi and M. S. Keane, Finitary orbit equivalence of odometers, Bull. London Math. Soc. 38 (2006), 450-458.

[HKR] T. Hamachi, M. S. Keane and M. K. Roychowdhury, Finitary orbit equivalence and measured Bratteli diagrams, Colloq. Math. 110 (2008), 363-382.

[HO] T. Hamachi and M. Osikawa, Ergodic groups of automorphisms and Krieger's theorems, Seminar Math. Keio Univ. 3 (1981).

[JS] A. del Junco and A. Sahin, Dye's theorem in the almost continuous category, preprint.

[KS1] M. Keane and M. Smorodinsky, Finitary isomorphism of irreducible Markov shifts, Israel J. Math. 34 (1979), 281-286.

[KS2] - - - A class of finitary codes, ibid. 26 (1977), 352-371.

[KS3] - - - Bernoulli schemes of the same entropy are finitarily isomorphic, Ann. of Math. 109 (1979), 397-406.

[ORW] D. Ornstein, D. Rudolph and B. Weiss, Equivalence of measure preserving transformations, Mem. Amer. Math. Soc. 262 (1982).

[R1] M. K. Roychowdhury, $\left\{m_{n}\right\}$-odometer and the binary odometer are finitarily orbit equivalent in: Ergodic Theory and Related Fields (Chapel Hill, NC, 20042006), I. Assani (ed.), Contemp. Math. 430, Amer. Math. Soc., 2007, 123-134.

[R2] - Irrational rotation of the circle and the binary odometer are finitarily orbit equivalent, Publ. RIMS Kyoto Univ. 43 (2007), 385-402.

[RR1] M. K. Roychowdhury and D. J. Rudolph, Any two irreducible Markov chains of equal entropy are finitarily Kakutani equivalent, Israel J. Math., to appear.

[RR2] -, 一, Any two irreducible Markov chains are finitarily orbit equivalent, ibid., to appear.

Department of Mathematics

Department of Mathematics

University of North Texas

Colorado State University

Denton, TX 76203, U.S.A.

Fort Collins, CO 80523, U.S.A.

E-mail: mrinal@unt.edu

E-mail: rudolphd@math.colostate.edu

Received 14 June 2007;

in revised form 6 November 2007 